\title{
A Novel “Work from Home Setup” for Telepathology Second Opinion of Oral Lesions
}

\author{
Nadeem Tanveer ${ }^{1}$ (D) $\cdot$ Neha Garg ${ }^{1}$
}

Received: 29 September 2020 / Accepted: 31 May 2021/Published online: 5 June 2021

(c) Indian Association of Surgical Oncology 2021

\begin{abstract}
The advent of smartphones with high-resolution cameras has opened up new possibilities in the field of telepathology for a second opinion especially in the COVID-19 era. The aim of this study was to evaluate the intraobserver concordance of a novel "work from home setup" with conventional microscopy diagnosis taken as the gold standard. A total of 90 cases of oral biopsies diagnosed by one pathologist were photographed with a smartphone camera. The static images were transferred via Google Drive and viewed on a 40-inch LED television screen connected by an HDMI cable to a laptop by the same pathologist. A wash-off period of 4 months was provided to avoid recall bias. The intraobserver concordance of Google Drive transfer and conventional microscopy was calculated. The cases in which the conventional slide microscopy and smartphone image diagnosis (SPID) via Google Drive were concordant constituted 98.9\% (89/90), and the ones with discordant diagnoses made up $1.1 \%$ (01/90). Smartphone-assisted telepathology can be useful for a second opinion. The images can be stored on a Google Drive and then downloaded to a personal computer and viewed on large-screen television to attain high levels of diagnostic accuracy.
\end{abstract}

Keywords Smartphone-assisted Telepathology $\cdot$ Google Drive transfer

\section{Introduction}

Moore's law promulgated by Gordon Moore in 1965 envisages that the number of transistors on a computing chip doubles every 18 months. The law still holds and the computing power of devices is improving at an exponential rate [1]. A corollary has been drawn with the improvement in smartphone camera resolution which has seen similar dramatic improvements in the last few years.

Pathologists have been quick to harness this improved camera resolution for smartphone-based telepathology. But, higher camera resolution comes at the cost of a larger size of the image file. This in turn requires faster internet connectivity and better viewing screens to effectively utilize the extra details available in a larger image. The availability of $4 \mathrm{G}$ networks has improved the internet speeds considerably;

Nadeem Tanveer

ntobh104@yahoo.co.in

Neha Garg

gargdoc118@gmail.com

1 Department of Pathology, University College of Medical Sciences, New Delhi 110095, India however, larger screens are generally not available to pathologists forced to work from home during the COVID-19 pandemic.

Large size light-emitting diode (LED) televisions are becoming more affordable and are now available in most households. For pathologists working from home, these televisions can be integrated with the laptops by a high-definition multimedia interface (HDMI) to provide an effective setup for telepathology for the second opinion.

Previous studies on smartphone-based telepathology have relied on either WhatsApp, email, or Google Drive to transfer the images and smartphone screens or desktops to visualize the images [2-5]. This is the first study utilizing the large-screen LED television screen to enhance the accuracy of smartphone-based telepathology with static images.

The utility of smartphone-based telepathology in remote areas with standalone laboratories and one or two pathologists is mainly for expert second opinions. However, even in bigger centers, it can be used for subspeciality second opinions where the specialist is on leave or in quarantine [6].

On March 26, 2020, Centres for Medicaid and Medicare Services issued a memorandum which allowed pathologists to review slides remotely during the COVID-19 pandemic 
in non-CLIA certified setups [7]. In April 2020, the FDA allowed the use of different digital pathology hardware and software even if they are not approved as medical devices [7].

\section{Aim}

To evaluate the intraobserver concordance of telepathology diagnosis of oral lesions using a novel setup with conventional microscopy diagnosis taken as gold standard.

\section{Material and Methods}

A total of 90 oral biopsies that had already been diagnosed on glass slide conventional microscopic examination by a single pathologist 4 months earlier were included in the study. The distribution of cases was as follows: 18 cases of leukoplakia, 62 cases of squamous cell carcinoma, 7 cases of severe dysplasia, and 3 cases of oral lichen planus. The images were photographed by another pathologist (with two and a half years of experience after post-graduation in pathology). She captured static images of slides from the ocular of a microscope (Dewinter classic microscope, Dewinter technologies, Milano, Italy) using a smartphone (One Plus A5000, 16 Megapixels, Made in Shenzen, Guangdong, China, By One Plus). Only the relevant areas were photographed and not the entire section. The details of the images captured are given in Table 1. One of the limitations of clicking images by a smartphone camera and viewing them on screen is the difficulty faced in orienting the images to one another. To help the pathologist orient the different images of the case, the different areas of the slide were marked with permanent markers of different colors-red, black, and brown. A low cost (\$10) commercially available smartphone camera adaptor (TechnoBuyers mobile phone adaptor) was used to stabilize the phone during image capture (Fig. 1).

Table 1 Basic data of the images captured by the smartphone camera

\begin{tabular}{lll}
\hline S. no & Parameter & Value \\
\hline 1 & Total number of cases & 90 \\
2 & Total number of images & 618 \\
3 & The average number of images captured per case & 6.86 \\
4 & Number of cases that required additional images & $1(1.1 \%)$ \\
& $\quad$ for diagnosis (N, \%) & \\
5 & Diagnoses of cases selected for the study & 18 \\
& a. Leukoplakia & 62 \\
& b. Squamous cell carcinoma & 07 \\
& c. Severe dysplasia & 03 \\
\hline
\end{tabular}

The images were sent to the same reporting pathologist who had diagnosed the cases on conventional microscopy by Google Drive transfer after an interval of 4 months (to ensure an adequate wash-off period to avoid recall bias). To make sure that the reporting pathologist was blinded to the diagnosis, all the cases were randomized. The reporting pathologist was given the option to ask for additional images at the same or higher magnification if needed.

Google Drive transfer was preferred over email transfer since it allowed the faster and efficient transfer of large image files. Clinical information such as age, type, and site of the lesion was also sent to the pathologist. A 40-inch large-screen LED television was attached by HDMI (highdefinition multimedia interface) cable to the laptop (Fig. 2A, $\mathrm{B}, \mathrm{C})$ and the images were viewed on the television screen for better accuracy (Fig. 2D, E, and Fig. 3A, B).

To avoid interobserver variability in the interpretation of the cases, the images were diagnosed by the same pathologist.

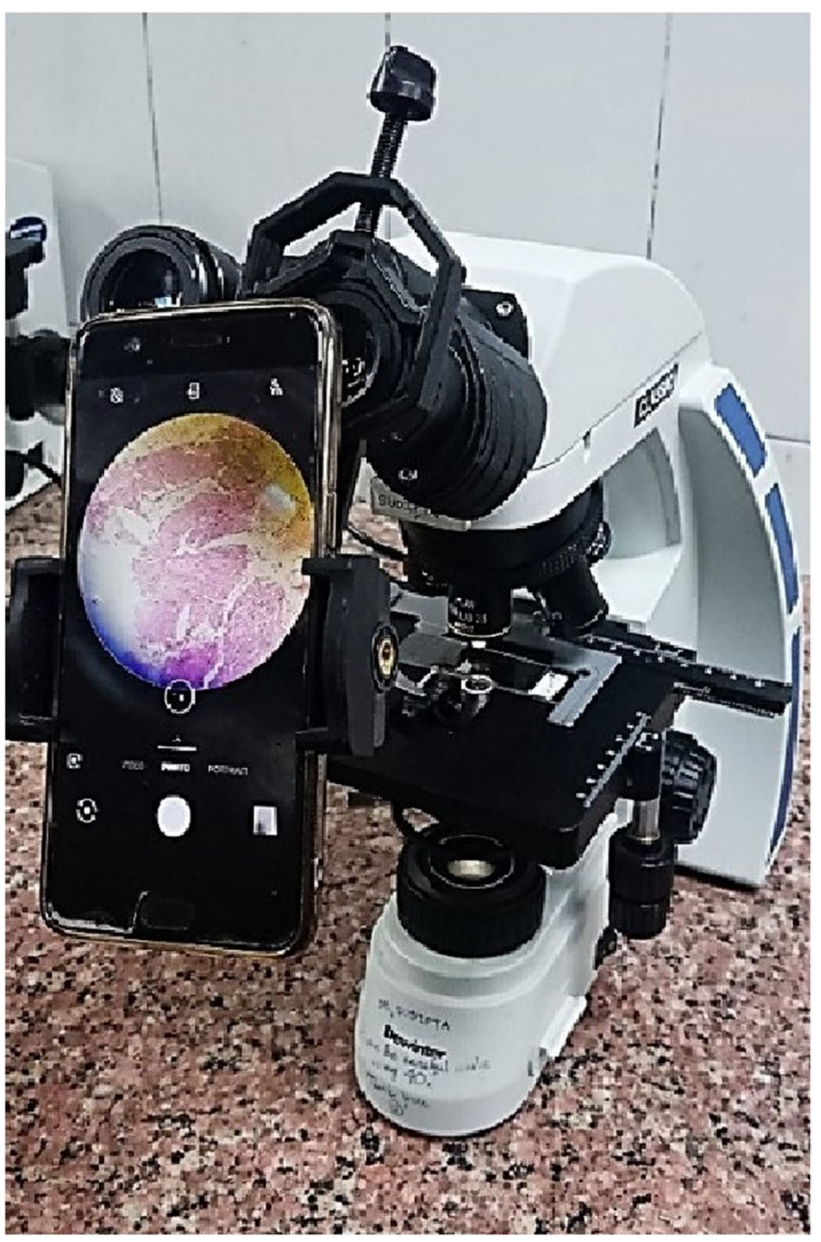

Fig.1 The technique of taking smartphone images of cases with an adaptor 
Fig.2 A The HDMI input option of the LED television set. B HDMI cable inserted into the HDMI port of the laptop. C Selecting multiple displays in the display settings of the laptop. D A case of leukoplakia seen on the LED television screen (hematoxylin and eosin stain $10 \times)$. E A case of welldifferentiated squamous cell carcinoma as seen on the bigger display (hematoxylin and eosin stain, $40 \times)$

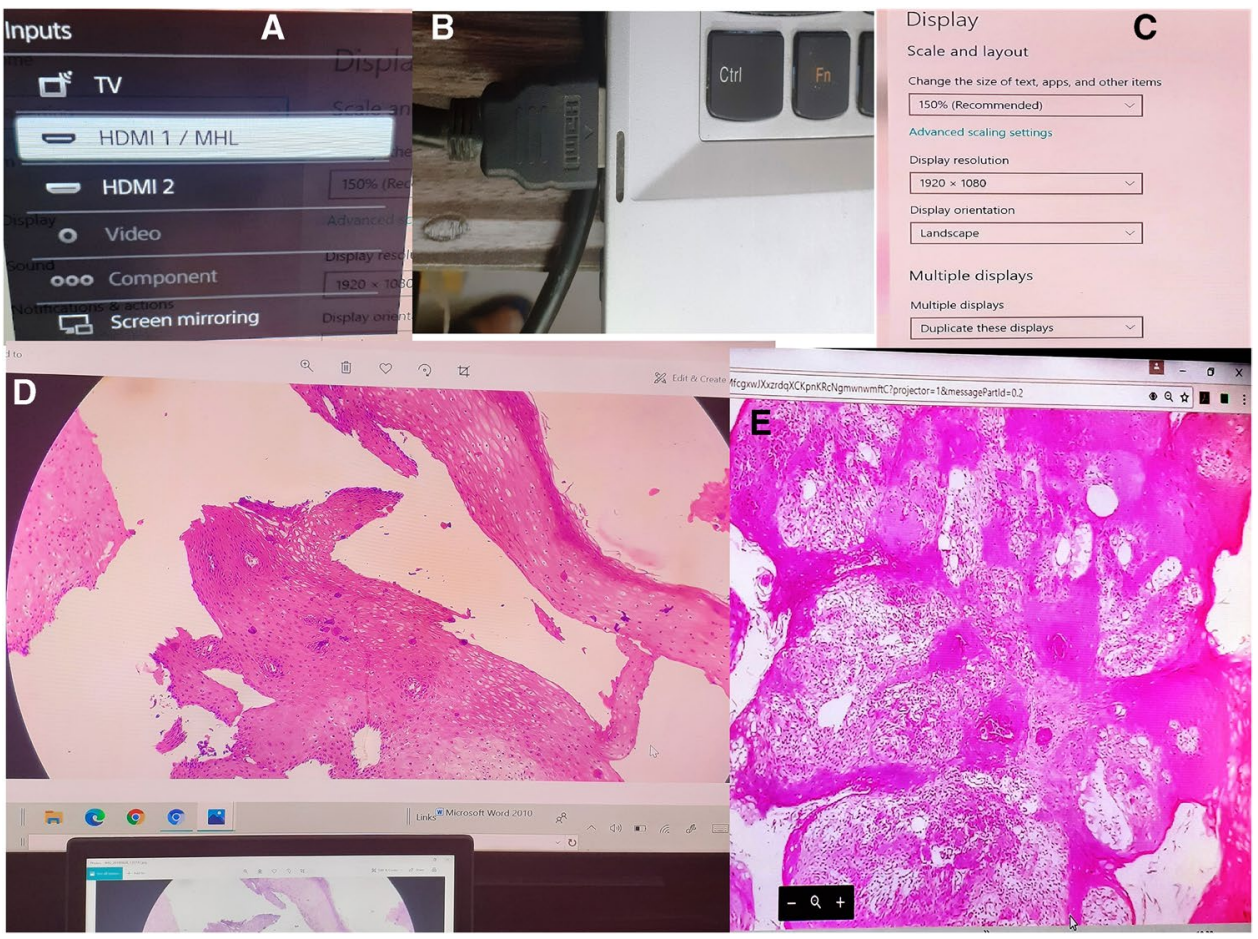

Microscopic diagnoses (MD) and SP image diagnoses (SPIDs) via Google Drive transfer were typed in the EXCEL sheet and statistical analysis was done using SPSS version 20 .

\section{Results}

The basic data for the images captured by the smartphone camera are given in Table 2. The mean image capture time for each case was $6.2 \mathrm{~min}$.

When the images were viewed on a large-screen 40-inch LED television screen attached to the laptop after Google Drive transfer, the concordance rate with conventional microscopy was $98.9 \%$ (89/90) and the discordance rate was $1.1 \%$ (1/90) (Table 3). All (62/62) cases of squamous cell carcinoma, all (18/18) cases of leukoplakia, and all (3/3) cases of oral lichen planus were correctly diagnosed after viewing the images on the large screen after Google transfer (Fig. 3A and B). Only one case (1/7) of severe dysplasia was overinterpreted as moderately differentiated squamous cell carcinoma (Table 3 ).

The discordant case was reviewed again both on conventional microscopy and on smartphone images to ascertain the reason for discordance. The possible reason for this discrepancy was the absence of a high power image of the representative area. This made the pathologist misinterpret invasion when there was none. With more experience with this technology, this type of error can be minimized.

\section{Discussion}

With the current COVID-19 crisis around the world, telepathology for the second opinion would play an important part in pathology practice [8]. There could be scenarios where the senior more experienced pathologist working in the lab is in-home quarantine and not available to see the slides. An inter-institutional second opinion from senior colleagues
Fig. 3 A and B Moderately differentiated squamous cell carcinoma (two cases) as seen on the television screen with laptop screen in the foreground for comparison (hematoxylin and eosin stain, $40 \times$ )

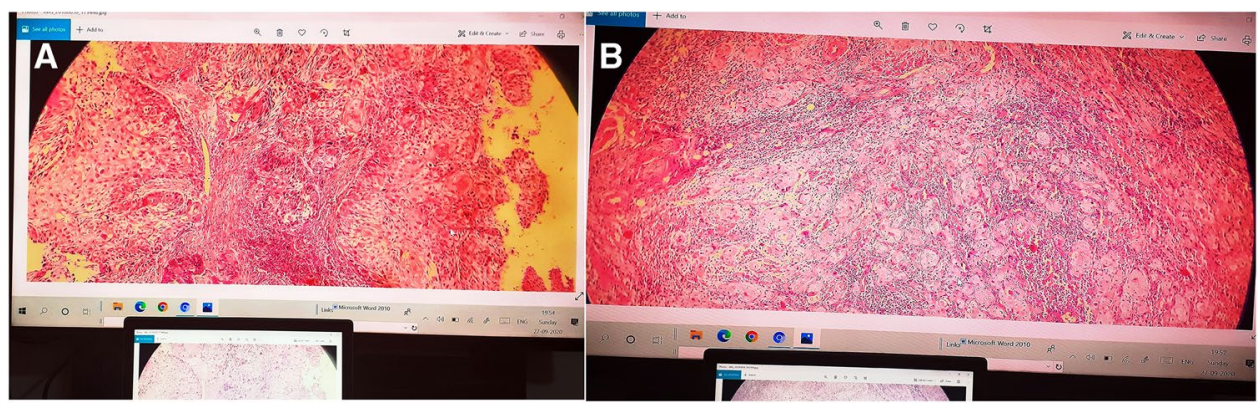


Table 2 Concordance rates for different categories of cases by Google Drive transfer

\begin{tabular}{lllll}
\hline S. no & Diagnosis & \multicolumn{2}{l}{$\begin{array}{l}\text { Google Drive transfer with the } \\
\text { LED television screen }\end{array}$} \\
\cline { 3 - 5 } & & $\begin{array}{l}\text { Total } \\
\text { Correctly } \\
\text { diagnosed }\end{array}$ & $\begin{array}{l}\text { Percentage } \\
\text { concord- } \\
\text { ance }\end{array}$ \\
\hline 1 & Leukoplakia & 18 & 18 & $100 \%$ \\
2 & Squamous cell Carcinoma & 62 & 62 & $100 \%$ \\
3 & Severe dysplasia & 7 & 6 & $85.7 \%$ \\
4 & Oral lichen planus & 3 & 3 & $100 \%$ \\
& Total & 90 & 89 & $98.9 \%$ \\
\hline
\end{tabular}

working in other labs would also be difficult with lockdowns in cities and telepathology can come to the rescue [7].

Telepathology may also be utilized in a limited way for tumor board meetings and second opinion [7]. The additional benefit of using telepathology is the reduced handling of histopathology slides and trays. Although in absolute terms, it is not clear whether it will reduce the spread of infection or not; however, it may reduce anxiety among the pathologists [8].

The use of WhatsApp for image transfer has the limitation of reduced image quality since it reduces the file size to facilitate rapid image transfer as also suggested by Garg et al. and Sarode et al. in their study [4, 5]. This limitation can be overcome by simple alternatives like renaming the image file as docx or pdf file, sending a zip file of images, uploading to the cloud, and sharing the link [4]. Another way to share high-quality big-size images is by uploading the images to Google Drive sharing the link. The receiver can download the images to his/her computer and view them. The size of the screen can be further increased by attaching a large-screen television by HDMI cable to the laptop.

Sarode et al. reported a correct second opinion diagnosis (SOD) for $95.26 \%$ of cases [5]. Garg et al. also reported an intraobserver concordance rate of $95 \%$ for WhatsApp-based telepathology for oral lesions [4]. The possible reasons for discordance in the diagnosis using WhatsApp transfer were the problem of orienting the different bits of the biopsy and pixelation of the image on digital zoom since the size of the image file is reduced on WhatsApp transfer. Pixelation can be overcome by a more efficient transfer of image with minimal loss of image detail.

With the availability of faster internet connections, the transfer of images has become easier. An interesting observation is that the download speeds of most connections are much faster than the upload speeds. So, the critical determinant of the functional ability of smartphone-based telepathology is the upload speed of the internet connection of the person asking for a second opinion. Some points to be taken care of while uploading the images are worth a mention. Too many background programs can take up much of the bandwidth. Syncing photos, backing up data to the cloud, and other file sharing can slow down the upstream connection. The upstream connection speeds can be tested with different devices. If it is significantly faster with one device, then there might be a hardware or software issue with the device rather than an internet problem. Updating the modem and router firmware can also help improve the speeds. If one is using a Wi-Fi connection, then staying close to the router and keeping the router at sufficient height can help.

The most efficient way to pair the laptop with the television is the HDMI cable. However, if the distance between the laptop and the television screen makes wires unrealistic, then wireless HDMI or Google Chromecast can be used. The other options include using a USB-C cable or a VGA cable depending on the ports available on the laptop or desktop computer.

The intraobserver concordance rate in our study was $98.9 \%$ and the discordance rate was $1.1 \%$ (Table 2) with the use of a large television screen after Google Drive transfer of images. The concordance rate is better than the concordance rates reported for similar studies using smartphone screens for viewing the images [2-5]. This could be explained by the visualization of images on a bigger screen with better resolution and no loss of image file size on Google Drive transfer.

A key determinant of acceptance of any new technology is the stakeholder's attitude. Recent surveys conducted among pathologists show a definite trend towards greater acceptance of the telepathology setup $[9,10]$. For pathologists who have spent a better part of their lives looking into microscopes, evaluating images on the screen is a big change which might take some time to be accepted. However, the

Table 3 The case given a different diagnosis by conventional microscopic evaluation and novel work from home set up using smartphone images via Google Drive transfer

\begin{tabular}{llll}
\hline S. no & Conventional microscopic diagnosis & $\begin{array}{l}\text { Smartphone image diagnosis (Google Drive } \\
\text { transfer) }\end{array}$ & The possible reason for discordance \\
\hline 1 & Severe dysplasia & $\begin{array}{c}\text { Moderately differentiated squamous cell carci- } \\
\text { noma }\end{array}$ & $\begin{array}{c}\text { The absence of a high power image of the } \\
\text { diagnostic area. This made the patholo- } \\
\text { gist misinterpret invasion when there } \\
\text { was none. }\end{array}$ \\
\hline
\end{tabular}


younger generation is already getting used to seeing smartphone images of cases on social media-WhatsApp and Twitter. Pathologists have taken to Twitter in a big way for discussing difficult cases, teaching, and connecting with experts. The large number of cases discussed on Twitter is a testament to the fact that pathologists are getting comfortable with telepathology [11, 12].

One limitation of the study was that only one pathologist was involved in the interpretation of telepathology images. The study would have been stronger if more than one pathologists were involved in the interpretation. One drawback of this technique of telepathology is the requirement of a trained pathologist to photograph the representative areas unlike digital scanners which can be operated by technicians. The advantage is that it can be set up at a reasonable cost with pre-existing LED television available in most households and by purchasing a low-cost smartphone adaptor.

The single case with discordant diagnosis highlights the problem of selection of representative area while taking the images with a smartphone camera. As the operators navigate the learning curve, this type of error would reduce.

To conclude, our study found that a bigger LED television screen attached to a laptop can provide a convenient setup for telepathology for a second opinion. Smartphone-assisted telepathology has shown promising results for the second opinion; however, for primary diagnosis, there is still a lot of ground to cover [4]. A similar setup would also be helpful in institutions and hospitals and also for teaching purposes.

\section{Declarations}

Ethical Approval This article does not contain any studies with human participants or animals performed by any of the authors since the study did not affect patient management or final diagnosis in any way institutional ethics board clearance was not required.

Informed Consent Informed consent was not needed as no human participants were included as subjects.

Conflict of Interest The authors declare no competing interests.

\section{References}

1. Khan HN, Hounshell DA, Fuchs ERH (2018) Science and research policy at the end of Moore's law. Nat Electron 1:14-21. https:// doi.org/10.1038/s41928-017-0005-9

2. Sahin D, Hacisalihoglu UP, Kirimlioglu SH (2018) Telecytology: is it possible with smartphone images? Diagn Cytopathol 46:40-46

3. Fontelo P, Liu F, Yagi Y (2015) Evaluation of a smartphone for telepathology: lessons learned. J Pathol Inform 6:35

4. Garg N, Tanveer N, Gaur JH (2019) Utility of WhatsApp as a tool for tele-oncopathology for oral lesions. Indian J Surg Oncol $10: 446-450$

5. Sarode SC, Sarode GS, Anand R, Patil S, Unadkat H (2017) WhatsApp is an effective tool for obtaining second opinion in oral pathology practice. J Oral Pathol Med 46:513-519

6. Bath NM, Wang X, Bledsoe JR, Thijssen M, Ahearn A, Movahedi B, Bozorgzadeh A, Martins PN (2018) The use of Smartphone for liver graft biopsy assessment at the time of procurement. Transplantation 102:e459-e460. https://doi.org/10.1097/TP.00000 00000002389

7. Henriksen J, Kolognizak T, Houghton T, Cherne S, Zhen D, Cimino PJ, Latimer CS, Scherpelz KP, Yoda RA, Alpers CE, Chhieng DF, Dirk Keene C, Gonzalez-Cuyar LF (2020) Rapid validation of telepathology by an academic neuropathology practice during the COVID-19 pandemic. Arch Pathol Lab Med. https://doi.org/ 10.5858/arpa.2020-0372-SA

8. Mukhopadhyay S, Booth AL, Calkins SM, Doxtader EE, Fine SW, Gardner JM, Gonzalez RS, Mirza KM, Jiang XS (2020) Leveraging technology for remote learning in the era of COVID-19 and social distancing: tips and resources for pathology educators and trainees. Arch Pathol Lab Med. https://doi.org/10.5858/arpa. 2020-0201-ED

9. Chordia TD, Vikey A, Choudhary AB, Samdariya Y, Chordia DS (2016) Current status and future trends in telepathology and digital pathology. J Oral Maxillofac Pathol 20:178-182

10. Gozali E, Safdari R, Sadeghi M, Saeidi MG, NiakanKalhori SR, Noroozinia F, Fazlollahi ZZ, Rahimi B (2017) Preconceived stakeholders' attitude toward telepathology: implications for successful implementation. J Pathol Inform 8:50

11. Fuller MY, Allen TC (2016) Let's have a Tweetup: the case for using Twitter professionally. Arch Pathol Lab Med 140:956-957

12. Isom J, Walsh M, Gardner JM (2017) Social media and pathology: where are we now and why does it matter? Adv Anat Pathol 24:294-303

Publisher's Note Springer Nature remains neutral with regard to jurisdictional claims in published maps and institutional affiliations. 